\title{
Expression of tumour necrosis factor $a$ and accumulation of fibronectin in coronary artery restenotic lesions retrieved by atherectomy
}

Nadine Clausell, Valter Correa de Lima, Silvana Molossi, Peter Liu, Eva Turley, Avrum I Gotlieb, Allan G Adelman, Marlene Rabinovitch

\begin{abstract}
Background-The formation of coronary artery neointima experimentally induced in piglets after cardiac transplantation is related to an immune-inflammatory reaction associated with increased expression of $T$ cells and inflammatory mediators (tumour necrosis factor $a$ and interleukin 1/) and upregulation of fibronectin. In vivo blockade of tumour necrosis factor $a$ in rabbits after cardiac transplantation results in reduced neointimal formation. The objective of this study was to investigate the hypothesis that coronary restenosis after atherectomy or percutaneous balloon angioplasty is associated with a similar inflammatory cascade initiated by mechanical injury.
\end{abstract}

Methods-Specimens taken at coronary atherectomy were analysed from 16 patients. Nine had had the procedure performed twice, firstly, to remove a primary lesion, and secondly, to remove a restenotic lesion. Seven had percutaneous balloon angioplasty after removal of restenotic tissue. Coronary atherectomy specimens were analysed by immunohistochemistry for the presence of T cells, macrophages, major histocompatibility complex II, interleukin $1 \beta$, tumour necrosis factor $\alpha$, fibronectin, and the receptor for hyaluronan mediated motility.

Results-The groups were clinically and angiographically similar with equivalent lumens before and after atherectomy. Restenotic lesions had increased expression of tumour necrosis factor $a$ and fibronectin compared with the primary lesions ( $P<0.05$ for both). There was also a trend towards a greater number of $T$ cells and increased expression of interleukin $1 \beta$.

Conclusions-Restenosis is associated with increased expression of tumour necrosis factor $a$ and fibronectin, suggesting that an immune-inflammatory reaction probably contributes to neointimal formation and may represent a form of wound healing and repair secondary to mechanical injury.

(Br Heart f 1995;73:534-539)

Keywords: inflammatory cells; cytokines; matrix; restenosis
The mechanisms underlying coronary artery restenosis after percutaneous transluminal procedures are poorly understood. Although smooth muscle cell proliferation may play a part, ${ }^{1}$ some reports suggest that it is not a prominent feature of the lesion. ${ }^{2}$ The role of other factors associated with vascular injury and repair, such as the production of extracellular matrix and inflammation, should therefore be considered in restenosis.

We found that intimal thickening in the ductus arteriosus is associated with smooth muscle cell migration, which is related to increased production of fibronectin and expression of the receptor for hyaluronan mediated motility. ${ }^{34}$ We also found that neointimal formation in piglet coronary arteries after cardiac transplantation is associated with increased endothelial and smooth muscle cell fibronectin synthesis induced by tumour necrosis factor $\alpha$ and interleukin $1 \beta$ after an immune inflammatory reaction..$^{5-7}$

We hypothesised that a similar immuneinflammatory mechanism may occur in coronary artery restenosis as a consequence of mechanical injury. In this study, we compared specimens taken at coronary atherectomy from a select group of patients who had the procedure twice-that is, for both primary and restenotic lesions. We also studied atherectomy specimens obtained from a clinically similar group of patients who had atherectomy for restenotic lesions after balloon angioplasty.

\section{Material and methods}

CLINICAL DATA RELATED TO DIRECTIONAL CORONARY ATHERECTOMY AND ANGIOGRAPHY Atherectomy was performed on the basis of clinical indications at the Toronto Hospital and Sunnybrook Medical Center in Toronto, Ontario, between July 1990 and August 1993 using the Simpson AtheroCath (Devices for Vascular Interventions, Redwood, CA). The protocol and informed consent for the atherectomy procedure were approved by the Human Ethics Review Committee of the Toronto Hospital, the coordinating centre. The 16 cases studied were retrospectively selected to include the cohort of nine patients who had had atherectomy performed twice: initially to treat a primary lesion and subsequently to treat a restenotic lesion. The seven other patients with restenosis who had atherectomy after balloon angioplasty were matched for age and clinical condition. The 
degree of coronary artery stenosis before atherectomy was assessed by quantitative coronary analysis using the cardiac measurement system (Medical Imaging Systems, Neunen, the Netherlands), as previously described. ${ }^{8}$

The cardiac medical history of the 16 patients was recorded with regard to the presence of coronary risk factors, clinical presentation of the ischaemic episodes (stable or unstable angina), site of the coronary artery lesions, and the time since the primary procedure. There was no uniform pharmacological regimen before or after the procedure.

\section{HISTOPATHOLOGY AND}

IMMUNOHISTOCHEMISTRY OF THE

ATHERECTOMY SPECIMENS

Atherectomy tissue was fixed in $10 \%$ formalin and embedded in paraffin. Sections were stained with haematoxylin and eosin and Movat pentachrome. The sections were analysed by two observers (AIG, VCL) who were unaware of the nature of the specimens-for example, whether they were primary or restenotic lesions. Sections showed the presence of plaque (intima) in all cases, media in most cases, and, rarely, adventitia. All analyses refer specifically to the plaque tissue. The presence of thrombosis was recorded. The extent of fibrosis and cellularity in the plaque was qualitatively graded as low, moderate, or high.

Deparaffinised sections were used for all immunoperoxidase staining procedures. To determine the presence of inflammatory cells and an immune reaction, monoclonal mouse antihuman antibodies to major histocompatibility complex class II antigens (1:10 dilution), $\mathrm{T}$ cells (CD45 RO) (1:50 dilution), and macrophages (leukocyte antigen L1) (1:50 dilution) (all immunoglobulin $G$ from Dakopatts Corporation, Carpinteria, CA) were used. To assess expression of the cytokines, interleukin $1 \beta$, and tumour necrosis factor $\alpha$ we used an affinity purified polyclonal rabbit antihuman immunoglobulin $\mathrm{G}$ interleukin $1 \beta$ antibody (1:200 dilution, Endogen, Boston, MA) and a monoclonal antihuman immunoglobulin $\mathrm{G}$ tumour necrosis factor $a$ antibody (1:200 dilution, ascites fluid, immunoglobulin $G_{1}$ from Sera-Lab, Crawley Down, Sussex). Fibronectin was identified using a monoclonal immunoglobulin $G_{1}$ antihuman cellular fibronectin antibody (1:500 dilution, ascites fluid, UBI, New York, NY) which does not recognise plasma fibronectin. Cells expressing the receptor for hyaluronan mediated motility were localised using a polyclonal antibody (1:1000 dilution). ${ }^{9}$ Positive characterisation of smooth muscle cells present in the tissue was performed using a monoclonal antibody to $a$ actin in a 1:400 dilution (immunoglobulin $\mathrm{G}_{2} \mathrm{a}$, Sigma, St Louis, MO). After incubation with the appropriate secondary antibodies, the sections were developed with 3,3'diaminobenzidine (Sigma). Control sections were treated with phosphate buffered saline alone or with appropriate normal isotypic immunoglobulin G (Dakopatts Corporation).

As previously described, ${ }^{5}$ the amount of each specific antigen in the plaque was semiquantitatively assessed (table) by three independent observers (NC, SM, MR) who were blinded as described above. The scoring system considered both the area of tissue with positive staining and the intensity of staining. A random sample of cases was also reviewed by a fourth investigator (AIG), independently, as an internal control mechanism. An overall agreement of greater than $80 \%$ of grading scores was achieved among the readers.

\section{STATISTICAL ANALYSIS}

Results are expressed as means (SE). Continuous variables of interest were assessed in primary and restenotic lesions by analysis of variance with post-hoc paired subgroup testing performed by Neuman-Keul's analysis that took into account the $\mathrm{P}$ value correction for multiple comparisons. The association between restenosis and categorical variables from the immunohistochemistry studies was analysed with Fisher's exact test. Differences were considered significant if $P \leqslant 0.05$.

\section{Results}

CLINICAL AND ANGIOGRAPHIC

CHARACTERISTICS (MEAN (SEM)) OF THE

PATIENTS

All 16 patients were men, they were of comparable age at the time of restenosis and primary atherectomy or percutaneous transluminal coronary angioplasty (56 (4) and 60 (4), respectively). The incidence of risk factors for coronary artery disease and unstable angina was similar. The time to reintervention for restenosis was not significantly different: 197 (34) days after atherectomy (median 210) and 107 (50) days after percutaneous transluminal coronary angioplasty (median 46$)(P=$ $0 \cdot 15)$, although it was longer after primary atherectomy because one patient in the group had reintervention at 390 days.

The degree of stenosis was similar when the primary atherectomy or percutaneous transluminal coronary angioplasty was performed $(67.4 \%(4 \cdot 0 \%)$ and $63.3 \%(3 \cdot 2 \%)$, respectively). The degree of restenosis after atherectomy was $67 \cdot 7 \%(4.5 \%)$ and after percutaneous transluminal coronary angioplasty $63.5 \%(4 \cdot 3 \%)$. The percentage of residual diameter stenosis after the atherectomy for restenosis was also similar: $26 \cdot 3 \%$ $(7 \cdot 4 \%)$ and $28 \cdot 9 \%(5 \cdot 2 \%)$, respectively.

\section{HISTOPATHOLOGY AND}

IMMUNOHISTOCHEMISTRY

There were no significant differences in the relative amounts of fibrosis or cellularity when comparing primary atherectomy tissue with the restenotic lesions after atherectomy and balloon angioplasty. The four cases in which thrombus was evident all presented with unstable angina (3/9 primary lesions and 1/7 restenotic lesions after balloon angioplasty). 


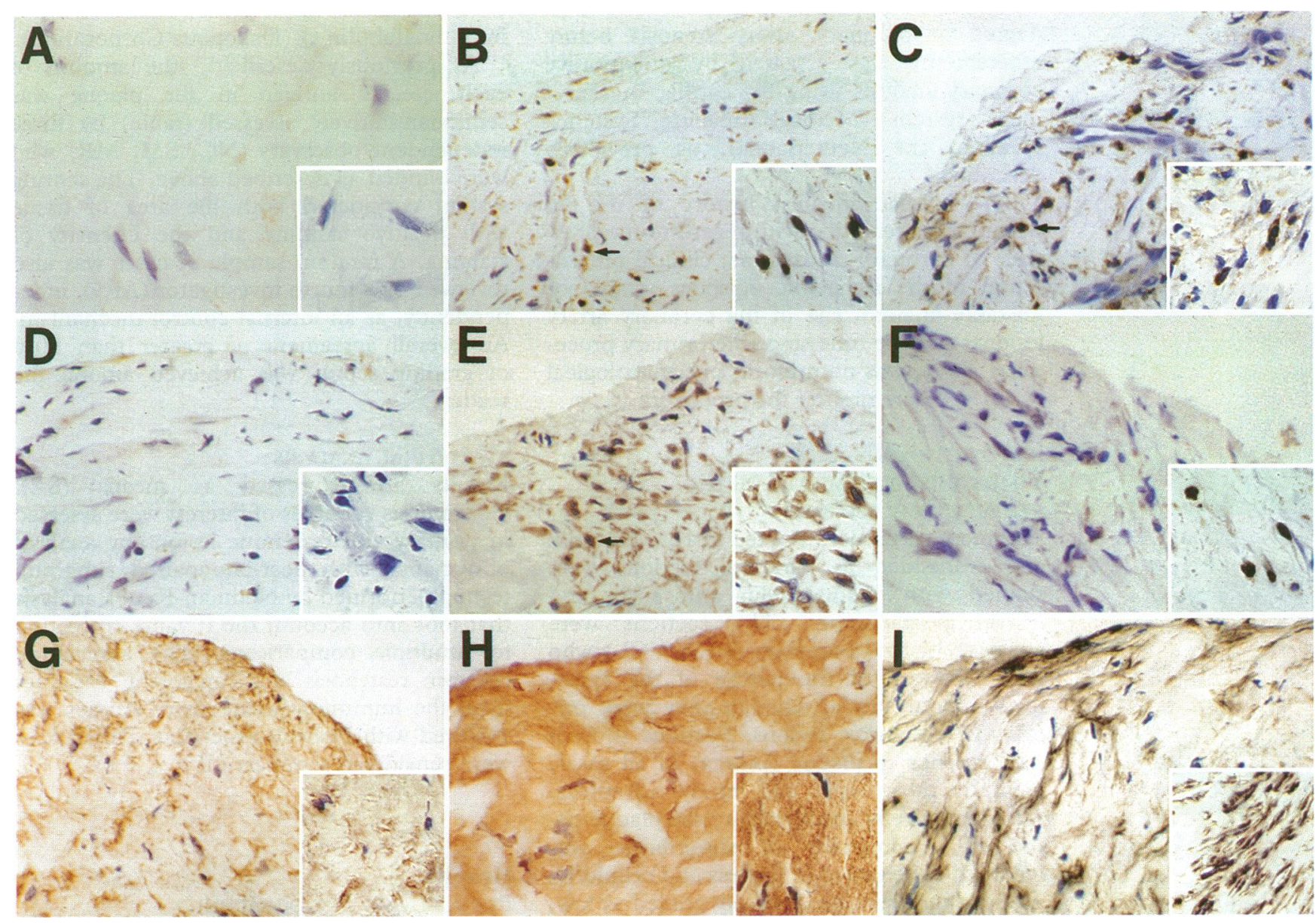

Immunoperoxidase staining for $T$ cells $(A, B, C)$, tumour necrosis factor $a(D, E, F)$, and fibronectin in specimens at coronary artery atherectomy from a primary lesion $(A, D, G)$, a restenotic lesion after atherectomy $(B, E, H)$ (both from the same patient) and a restenotic lesion after balloon angioplasty $(C, F, I)$. Negative $T$ cell staining was observed in the primary lesion $(A)$, whereas several positive cells were observed in both restenotic lesions $(B$, $C)$. Similarly, more intense staining for tumour necrosis factor a was observed in the restenotic lesions $(H, I)$ both cellularly and extracellularly than in the primary lesion $(G)$. The expression of tumour necrosis factor a seemed to be associated with inflammatory cells and smooth muscle cells as judged by results on light microscopy. Mild staining for fibronectin was observed in the primary lesion $(G)$, whereas more intense staining was observed in both restenotic lesions $(H, I)$. Original magnification $\times 40$ with $\times 100$ under oil immersion for all insets. Note: arrows are examples of positive staining for peroxidase.

Thrombus was not evident, however, in seven additional patients with unstable angina, but fresh microthrombi might have been lost during the procedure or on retrieval of tissue, or both.

The pattern of immunostaining observed for major histocompatibility complex class II antigens was similar in all specimens. The numbers of $T$ cells were increased in restenotic lesions compared with primary lesions. None of the nine cases of primary lesions showed more than mild staining, whereas in five of the 16 cases of restenosis (3/9 after atherectomy and 2/7 after percutaneous transluminal coronary angioplasty), showed moderate to intense staining. Specimens from six of the nine patients who had repeat atherectomy showed increased staining for $T$ cells in the restenotic lesions compared with the primary lesions. Only one of the nine primary lesions showed more than mild staining for macrophages, whereas four of the 16 cases of restenosis (two out of nine after atherectomy and two out of seven after percutaneous transluminal coronary angioplasty) showed moderate to intense staining. Also, in four of the nine patients who had repeat atherectomy macrophages stained darker in the restenotic lesions than in the pri- mary lesions. In all specimens examined, the pattern of immunostaining for $T$ cells and macrophages was mostly focal (figure and table).

Staining for tumour necrosis factor $a$ was significantly higher ( $>$ mild) in restenotic than in primary lesions $(P<0.05)$. Only one out of nine primary lesions showed more than mild staining for tumour necrosis factor $a$, whereas nine out of 16 cases of restenosis (4/9 after atherectomy and 5/7 after percutaneous transluminal coronary angioplasty) had moderate to intense staining. In patients who had repeat atherectomy, staining for tumour necrosis factor $a$ was more intense in seven out of nine restenotic lesions than in the primary lesions (figure and table).

Immunoperoxidase staining for interleukin $1 \beta$ was no more than mild in all of the primary lesions, whereas five out of 16 cases of restenosis (4/9 after atherectomy and 1/7 after percutaneous transluminal coronary angioplasty) showed moderate to intense staining. Four of the nine patients who had repeat atherectomy showed greater immunoperoxidase staining for interleukin $1 \beta$ in the restenotic lesions than in the primary lesions. In all of the specimens studied the pattern of immunostaining for interleukin $1 \beta$ and 
Summary of immunohistochemical features according to lesion type

\begin{tabular}{|c|c|c|c|c|c|c|c|}
\hline Case $\mathrm{No}^{\star}$ & $T$ cells & Macrophages & $\begin{array}{l}\text { Major } \\
\text { histocompatibility } \\
\text { complex }\end{array}$ & $\begin{array}{l}\text { Tumor } \\
\text { necrosis } \\
\text { factor a }\end{array}$ & Interleukin $1 \beta$ & Fibronectin & $\begin{array}{l}\text { Receptor for } \\
\text { hyaluronan } \\
\text { motility marker }\end{array}$ \\
\hline \multicolumn{8}{|c|}{ Primary lesion } \\
\hline 1 & \pm & - & - & \pm & - & ++ & - \\
\hline 2 & - & \pm & - & + & - & ++ & - \\
\hline 3 & \pm & + & - & \pm & - & + & + \\
\hline 4 & - & - & - & \pm & - & - & - \\
\hline 5 & - & \pm & - & - & - & + & - \\
\hline 6 & + & - & - & \pm & - & \pm & - \\
\hline 7 & \pm & + & - & + & - & + & - \\
\hline 8 & - & - & \pm & + & + & \pm & - \\
\hline 9 & + & ++ & - & ++ & + & + & \pm \\
\hline \multicolumn{8}{|c|}{ Restenosis after directional atherectomy } \\
\hline 10 & - & + & - & + & - & + & - \\
\hline 11 & + & - & - & ++ & - & ++ & \pm \\
\hline 12 & ++ & - & - & ++ & - & ++ & - \\
\hline 13 & + & ++ & - & + & - & ++ & \pm \\
\hline 14 & +++ & ++ & + & +++ & +++ & & +++ \\
\hline 15 & +++ & \pm & - & + & ++ & +++ & ++ \\
\hline 16 & - & \pm & - & + & - & +++ & - \\
\hline 17 & \pm & - & - & + & + & ++ & \pm \\
\hline 18 & - & \pm & - & +++ & +++ & \pm & - \\
\hline \multicolumn{8}{|c|}{ Restenosis after percutaneous transluminal coronary angioplasty } \\
\hline 19 & \pm & +++ & - & - & - & ++ & \pm \\
\hline 20 & + & - & - & - & - & + & - \\
\hline 21 & + & + & - & +++ & + & ++ & + \\
\hline 22 & ++ & + & \pm & +++ & ++ & ++ & ++ \\
\hline 23 & + & - & - & ++ & \pm & ++ & - \\
\hline 24 & ++ & ++ & - & +++ & \pm & + & \pm \\
\hline 25 & ++ & + & - & ++ & + & ++ & + \\
\hline
\end{tabular}

*Cases No 1 and 10,2 and 11,3 and 12,4 and 13,5 and 14,6 and 15, 7 and 16,8 and 17,9 and 18 are lesions from patients directional coronary atherectomy twice.

Grading system: $(-)$, negative; $( \pm)$, minimal; $(+)$, mild; $(++)$, moderate; $(+++)$, high.

tumour necrosis factor $a$ seemed to be associated with smooth muscle cells, inflammatory cells, and the extracellular matrix (table).

Immunostaining for fibronectin was significantly greater in restenotic than in primary lesions $(P<0.05)$. Only two of the nine primary lesions showed more than mild staining, whereas 11 of the 16 restenotic lesions (6/9 after atherectomy and 5/7 after percutaneous transluminal coronary angioplasty) showed moderate to intense staining. In five of the nine patients who had repeat atherectomy the restenotic lesions stained more strongly for fibronectin than did the primary lesions. In all specimens the pattern of fibronectin accumulation observed was both diffuse and focal and present in areas of both low and high cellularity (figure and table).

None of the nine primary lesions showed more than mild staining for the receptor for hyaluronan mediated motility, whereas three of the 16 restenotic lesions (2/9 after atherectomy and 1/7 after percutaneous transluminal coronary angioplasty) had moderate to intense staining. The pattern of staining for the receptor for hyaluronan mediated motility was usually focal, associated with cells which seemed to be both smooth muscle cells (positive staining for $a$ actin; especially obvious in those with a motile elongated phenotype) and inflammatory cells (as judged by cell markers previously described), but the receptor for hyaluronan mediated motility was also evident in the extracellular matrix (table).

\section{Discussion}

In this study of restenosis we had the opportunity to compare primary and restenotic lesions from patients who had had atherectomy twice, as well as restenotic lesions from patients who had percutaneous transluminal coronary angioplasty. Although the number of patients was small, comparison of tissue from the same patients before and after restenosis circumvents some of the difficulties related to individual variability. We found a significant increase in the presence of the inflammatory mediator tumour necrosis factor $a$ and in the accumulation of the extracellular matrix component fibronectin. Increases in immunostaining for other markers of inflammation, $T$ cells, macrophages, and interleukin $1 \beta$ and for the receptor for hyaluronan mediated motility were observed in both types of restenotic lesions, although significance was not achieved. This may reflect the small number of patients studied, the fact that only one point in time in this dynamic process was assessed, and that other pathophysiological mechanisms are important. Although technically inevitable, another potential limitation of this study may be related to the fact that atherectomy specimens may include not only restenotic tissue but also part of the adjacent primary lesion. This was less of a confounding factor in the group of patients who had had atherectomy twice and served as their own controls.

Different forms of vessel wall injury can induce adhesion to the endothelium of peripheral blood monocytes and $\mathrm{T}$ cells, which subsequently invade the artery wall. ${ }^{1011}$ Whether these cells actively cause the vascular lesion at a specific point in time may be difficult to assess, ${ }^{12}$ but the fact that, in our study, the presence of cytokines (major $\mathrm{T}$ cell products) also seemed to be increased in restenotic lesions would indirectly indicate that, at one stage, $T$ cells were modulating an inflammatory-like cell response. Once present in vascular lesions, $T$ cells can modulate the biological activity of endothelial and smooth muscle 
cells through expression of cytokines and growth factors. ${ }^{1314}$

Both increased tumour necrosis factor $\alpha$ and interleukin $1 \beta$ observed in restenotic lesions associated with $T$ cells and macrophages, as well as smooth muscle cells, may have a pathophysiological role in influencing the development of vascular lesions ${ }^{1215}$ through paracrine or autocrine mechanisms. ${ }^{6714}$ Our most recent experimental studies on the graft coronary arteriopathy have suggested that there is reciprocal coinduction of tumour necrosis factor $a$ and interleukin $1 \beta^{16}$ and that both cytokines upregulate endothelial and smooth muscle cell fibronectin synthesis. ${ }^{6717}$ Moreover, we showed in vivo, that neutralisation of tumour necrosis factor $a$ activity in rabbits after cardiac transplantation reduced both the severity and number of coronary artery lesions and this was associated with less inflammation and reduced accumulation of fibronectin in the vessel wall. ${ }^{18}$ Although we did not perform concurrent double labelling techniques to confirm the source of tumour necrosis factor $a$ because of scarcity of tissue, light microscopy showed that macrophages primarily and lymphocytes and smooth muscle cells to some extent are involved in the expression of this cytokine.

The increased cell associated fibronectin observed in restenotic lesions may be related to medial smooth muscle cell migration since fibronectin can form a gradient that promotes cell movement. ${ }^{19}$ The expression of the receptor for hyaluronan mediated motility in restenotic lesions may also be a marker of heightened cell migration. Increased expression of the receptor for hyaluronan mediated motility was seen at the tips of lamellipodia of advancing cells, and polyclonal antireceptor for hyaluronan mediated motility antibodies blocks cell motility. ${ }^{20}$ In our previous studies increased migration of ductus arteriosus smooth muscle in vitro was dependent on increased production of fibronectin and increased expression of receptor for hyaluronan mediated motility. ${ }^{4}$ Accumulation of fibronectin occurs even in early atherosclerotic lesions ${ }^{21}$ and in piglet coronary arteries in relation to cardiac allograft arteriopathy. ${ }^{5}$

Increased fibronectin may also induce transendothelial migration and subendothelial positioning of inflammatory cells in restenosis. This is related to the binding of $a 4 \beta 1$ and $a 5 \beta 1$ integrins to fibronectin at the connecting segment- 1 and the arginine-glycine-aspartate sites respectively. ${ }^{22}$ Our recent in vitro observations using an endothelial and smooth muscle cell coculture system support the functional significance of fibronectin production mediated by cytokines in the transendothelial migration of lymphocytes. ${ }^{23}$ We further showed that in vivo blockade of fibronectin interaction with $\mathrm{T}$ lymphocytes significantly reduced coronary artery neointimal formation in rabbit cardiac allografts. ${ }^{24}$

In summary, our observations suggest that restenotic lesions represent, at least in part, a form of wound healing and repair charac- terised by the presence of inflammatory cells, expression of cytokines (tumour necrosis factor $a$ and interleukin $1 \beta$ ), and accumulation of fibronectin. Although our observations characterise an immune-inflammatory mechanism in the pathogenesis of restenosis, recent studies have implicated cytomegalovirus and inhibition of p53 activity in the proliferative response in this process. ${ }^{25}$ Thus, restenosis probably is a multifactorial cascade affecting different mechanisms at different levels. On the basis of our observations therapeutic strategies that block cytokine function or inhibit fibronectin-lymphocyte interactions could potentially interfere with one of the steps in the progression of restenosis.

We thank Beverley Wu and Ken Ekam from the Department of Pathology of the Toronto Hospital (General Division) for processing the histological sections and Peter Lewyecky from the Division of Cardiology (Clinical Trials Unit) for statistical support. We thank Mike Starr from the department of pathology for his expert help with the colour photomicrographs and Joan Jowlabar and Susy Taylor from the Division of Cardiovascular Research, Hospital for Sick Children, for secretarial help. We also thank our colleagues Eric A Cohen and Brian P Kimball, who also performed atherectomy procedures and allowed us to study the material retrieved. This work was and allowed us to study the material retrieved. This work was supported Medical Research Council of Canada awarded to MR, by grants from the Medical Research Council of Canada to PL and AGA, and by a grant from the Heart and Stroke Foundation of Ontario (T-2351) to AIG. NC is a fellow, SM is supported by the Heart and Stroke Foundation of Ontario.

1 Simons M, Leclerc G, Safian R, Isner J, Weir L, Baim D Relation between activated smooth-muscle cells in coronary-artery lesions and restenosis after atherectomy. coronary-artery lesions and reste

2 O'Brien E, Alpers C, Stewart D, Ferguson M, Tran N, Gordon $\mathrm{D}$, et al. Proliferation in primary and restenotic coronary atherectomy tissue. Implications for antiproliferative therapy. Circ Res 1993;73:223-31.

3 Boudreau N, Rabinovitch M. Developmentally regulated changes in extracellular matrix in endothelial and smooth muscle cells in the ductus arteriosus may be related to intimal proliferation. Lab Invest 1991;64: 187-99.

4 Boudreau N, Turley E, Rabinovitch M. Fibronectin, hyaluronan and a hyaluronan binding protein contribute to increased ductus arteriosus smooth muscle cell migration. Dev Biol 1991;143:235-47.

5 Clausell N, Molossi S, Rabinovitch $M$. Increased interleukin- $1 \beta$ and fibronectin expression are early features of the development of the post-cardiac transplant coronary the development of the post-cardiac transplant coronary

6 Clausell N, Rabinovitch M. Upregulation of fibronectin synthesis by interleukin-1 $\beta$ in coronary artery smooth synthesis by interleukin- $1 \beta$ in coronary artery smooth
muscle cells is associated with the development of the muscle cells is associated with the development of the
post-cardiac transplant arteriopathy in piglets. $f$ Clin post-cardiac transplant

7 Molossi S, Clausell N, Rabinovitch M. Coronary artery endothelial interleukin-1 $\beta$ mediates enhanced fibronectin production related to post-cardiac transplan arteriopathy in piglets. Circulation 1993;88:248-56.

8 Adelman A, Cohen E, Kimball B, Bonan R, Ricci D, Webb J, et al. A comparison of directional atherectomy with balloon angioplasty for lesions of the left anterior descending coronary artery. $N$ Engl $₹$ Med 1993;329. 228-33.

9 Samuel S, Hurta R, Spearman M, Wright J, Turley E, Greenberg A. TGF- $\beta 1$ stimulation of cell locomotion utilizes the hyaluronan receptor RHAMM and hyaluronan. $\mathcal{H}$ Cell Biol 1993;123:749-58.

10 Ross R. The pathogenesis of atherosclerosis: a perspective for the 1990s. Nature 1993;362:801-9.

11 Walpola P, Gotlieb A, Langille B. Monocyte adhesion and changes in endothelial cell number, morphology, F-actin changes in endothelial cell number, morphology, F-actin
distribution elicited by low shear stress in vivo. Am $f$ Pathol 1993;142:1392-400.

12 Libby $P$, Hansson G. Involvement of the immune system in human atherogenesis: current knowledge and unanin human atherogenesis: current knowledge

13 Libby $P$, Warner S, Friedman G. Interleukin 1: a mitogen for human vascular smooth muscle cells that induces the release of growth-inhibitory prostanoids. $\mathcal{f}$ Clin Invest 1988;81:487-98.

14 Loppnow H, Libby P. Functional significance of human vascular smooth muscle cell-derived interleukin 1 in paracrine and autocrine regulation pathways. Exp Cell Res 1992;198:283-90

15 Jäättela M. Biologic activities and mechanisms of action of tumour necrosis factor- $\alpha /$ cachectin. Lab Invest 1991;64: 724-42. 
16 Molossi S, Clausell N, Rabinovitch M. Reciprocal induction of tumor necrosis factor- $\alpha$ and interleukin- $1 \beta$ activity mediates fibronectin synthesis in coronary artery smooth muscle cells. F Cell Physiol 1995;163:19-29.

17 Clausell N, Molossi S, Rabinovitch M. Tumor necrosis factor-alpha induction of smooth muscle cell fibronectin factor-alpha induction of smooth muscle cell fibronectin
synthesis via interleukin-1 $\beta$ is associated with the development of graft atherosclerosis. [Abstract.] Circulation 1993;88:I419.

18 Clausell N, Molossi S, Sett S, Rabinovitch M. In vivo blockade of tumor necrosis factor- $\alpha$ in cholesterol-fed rabbits following cardiac transplant inhibits acute coronary artery neointimal formation. Circulation 1994;89: 2768-79.

19 Linask K, Lash J. Precardiac cell migration: fibronectin localization at mesoderm-endoderm interface during directional movement. Dev Biol 1986;114:87-101.

20 Hardwick C, Hoare K, Owens R, Hohn H, Hook M, Moore $\mathrm{D}$, et al. Molecular cloning of a novel hyaluronan receptor that mediate

21 Shekhonin BV, Domogatsky SP, Idelson GL, Koteliansky
VE, Rukosuev VS. Relative distribution of fibronectin and type I, III, IV, V collagens in normal and atherosclerotic intima of human arteries. Atherosclerosis 1987;67: 9-16.

22 Ager A, Humphries M. Use of synthetic peptides to probe lymphocyte-high endothelial cell interactions. Lymphocytes recognize a ligand on the endothelial surface which contains the CS1 adhesion motif. Intern Immunol 1991;2:921-8.

23 Molossi S, Elices M, Rabinovitch M. Blockade of interleukin- $1 \beta$-induced fibronectin/lymphocyte interaction in vitro inhibits lymphocyte transendothelial migration. [Abstract.] FASEB f 1994;8:A1018.

24 Molossi S, Elices M, Arrhenius T, Diaz R, Coulber C, Rabinovitch $M$. Blockade of very late antigen-4 integrin binding to fibronectin with connecting segment-1 peptide reduces accelerated coronary arteriopathy in rabbit cardiac allografts. $f$ Clin Invest 1995 (in press).

25 Speir E, Modali R, Huang E, Leon M, Shawl F, Finkel T, et al. Potential role of human cytomegalovirus and p53 interaction in coronary restenosis. Science 1994;265: 391-4. 